\title{
Power Efficiency Improvements through Peak-to-Average Power Ratio Reduction and Power Amplifier Linearization
}

\author{
Ning Chen, ${ }^{1}$ G. Tong Zhou, ${ }^{2}$ and Hua Qian ${ }^{3}$ \\ ${ }^{1}$ Freescale Semiconductor, Inc., Austin, TX 78729, USA \\ ${ }^{2}$ School of Electrical and Computer Engineering, Georgia Institute of Technology, Atlanta, GA 30332, USA \\ ${ }^{3}$ Marvell Semiconductor, Inc., Santa Clara, CA 95054, USA
}

Received 9 June 2005; Revised 14 February 2006; Accepted 24 November 2006

Recommended by Enis Ahmet Cetin

\begin{abstract}
Many modern communication signal formats, such as orthogonal frequency-division multiplexing (OFDM) and code-division multiple access (CDMA), have high peak-to-average power ratios (PARs). A signal with a high PAR not only is vulnerable in the presence of nonlinear components such as power amplifiers (PAs), but also leads to low transmission power efficiency. Selected mapping (SLM) and clipping are well-known PAR reduction techniques. We propose to combine SLM with threshold clipping and digital baseband predistortion to improve the overall efficiency of the transmission system. Testbed experiments demonstrate the effectiveness of the proposed approach.
\end{abstract}

Copyright (c) 2007 Ning Chen et al. This is an open access article distributed under the Creative Commons Attribution License, which permits unrestricted use, distribution, and reproduction in any medium, provided the original work is properly cited.

\section{INTRODUCTION}

Modern transmission formats, such as orthogonal frequency-division multiplexing (OFDM) and code-division multiple access (CDMA), have gained tremendous popularity thanks to their high spectral efficiency. However, a drawback is the low power efficiency of these systems. OFDM and CDMA signals suffer from high peak-to-average power ratios (PARs), making them susceptible to nonlinearities that are inherent in the RF/microwave power amplifiers (PAs). To avoid nonlinear distortions, the average operating power of the PA has to be backed-off significantly, giving rise to low DC to RF conversion efficiency.

PA efficiency enhancement is a critical issue for wireless communication applications. In a typical cellular base station, the RF PA and its associated cooling equipment are responsible for approximately $50 \%$ of the overall DC power consumption and $60 \%$ of its physical size [1]. On the other hand, it is reported that in today's cellular phones, over $90 \%$ of the power used to transmit the signal is wasted in the form of heat that stays inside the phone [2]. The topic of power efficiency has attracted much attention in recent years.

There are two key factors that contribute to the low PA efficiency in these applications: (i) high PAR value of the signal, and (ii) nonlinearity of the PA. Many techniques have been proposed to reduce the PAR, such as deliberate clip- ping, complementary coding, selected mapping (SLM), and so forth [3-5]. Among the many PA linearization techniques, adaptive digital baseband predistortion is the most costeffective [6]. To the best of our knowledge, few references except for $[7,8]$ have discussed joint PAR reduction and PA linearization. In [7], the authors investigated the BER performance degradation due to inaccuracy of the side information of the PAR reduction in a multicarrier CDMA system, but gave no details of PA linearization. In [8], a commercial chip that implements deliberate clipping was used as the PAR reduction preprocessor and a lookup table was used for PA linearization. In this paper, we will (i) delineate the relationship between PAR reduction and PA linearization with respect to their contributions to power efficiency improvements; (ii) propose a modified SLM with thresholding and clipping technique and present a closed-form expression for the distribution of the PAR of the resulting signal; (iii) quantify the power efficiency enhancement in terms of increase in the average transmit power while keeping the adjacent channel power ratio (ACPR) fixed. We will demonstrate our approach through testbed experiments.

\section{POWER EFFICIENCY IMPROVEMENT CONCEPTS}

Consider the input-output characteristic of a PA shown in Figure 1(a). If we denote the baseband PA input by $x(t)$, 


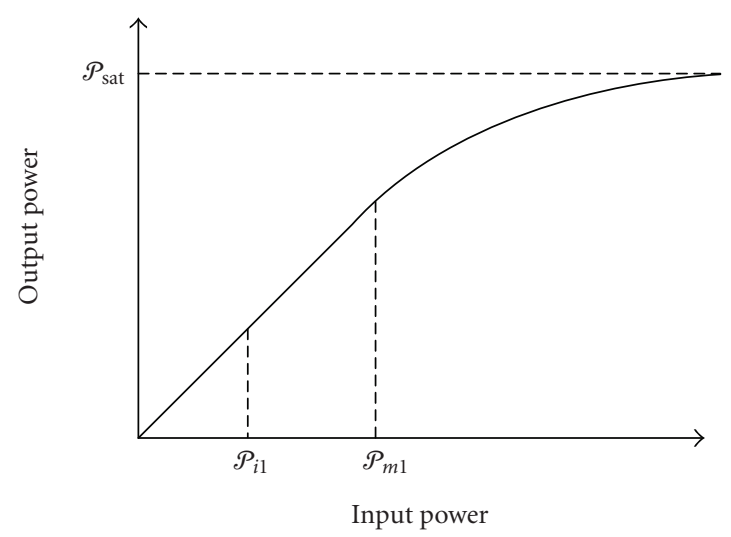

(a) Nonlinear PA with input backoff. $\operatorname{PAR}_{1}(\mathrm{~dB})=$ $\mathcal{P}_{m 1}(\mathrm{~dB})-\mathcal{P}_{i 1}(\mathrm{~dB})$.

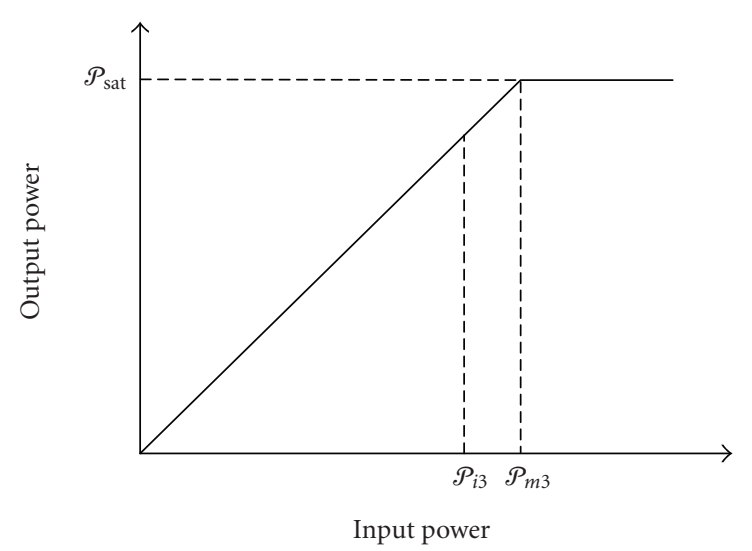

(c) After PAR reduction. PAR $_{3}(\mathrm{~dB})=\mathcal{P}_{m 3}(\mathrm{~dB})-\mathcal{P}_{i 3}(\mathrm{~dB})$. $\mathrm{PAR}_{3}<\mathrm{PAR}_{2} . \mathcal{P}_{m 3}=\mathcal{P}_{m 2}, \mathcal{P}_{i 3}>\mathcal{P}_{i 2}$.

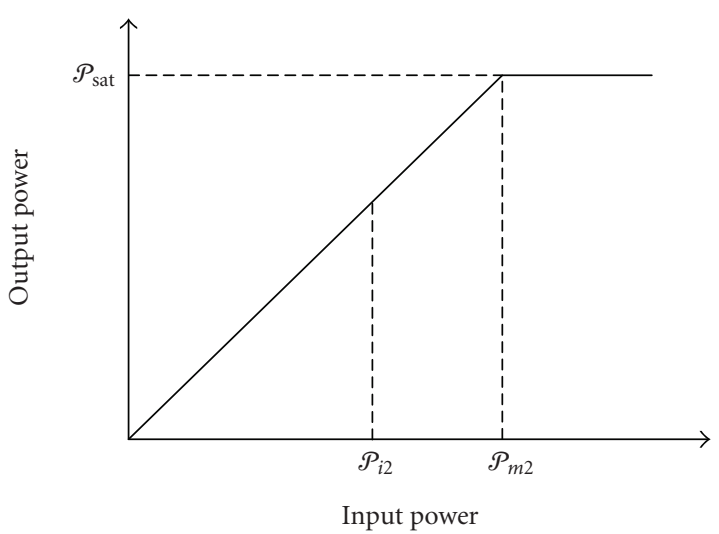

(b) Ideal linear PA. PAR $2(\mathrm{~dB})=\mathcal{P}_{m 2}(\mathrm{~dB})-\mathcal{P}_{i 2}(\mathrm{~dB})$ $\mathrm{PAR}_{2}=\mathrm{PAR}_{1} \cdot \mathcal{P}_{m 2}>\mathcal{P}_{m 1}, \mathcal{P}_{i 2}>\mathcal{P}_{i 1}$.

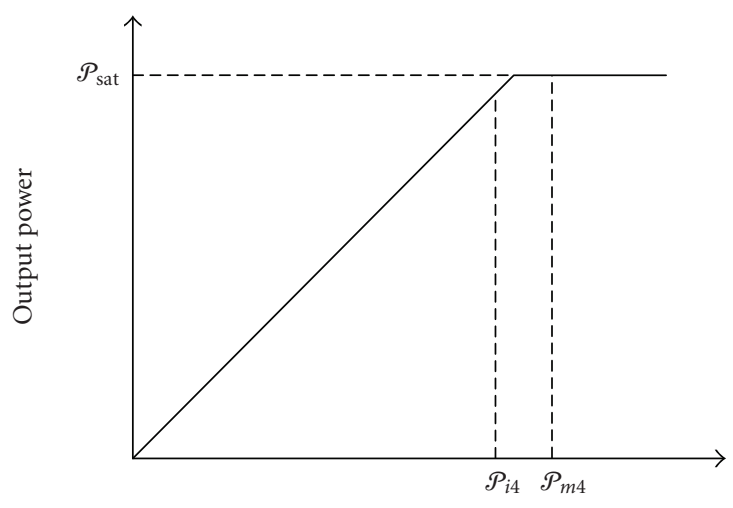

Input power

(d) Allow occasional saturation (clipping). $\mathrm{PAR}_{4}(\mathrm{~dB})=$ $\mathcal{P}_{m 4}(\mathrm{~dB})-\mathcal{P}_{i 4}(\mathrm{~dB}) . \mathrm{PAR}_{4}=\mathrm{PAR}_{3} . \mathcal{P}_{m 4}>\mathcal{P}_{m 3}, \mathcal{P}_{i 4}>\mathcal{P}_{i 3}$

FIgURe 1: PA linearization and PAR reduction can improve the PA efficiency by reducing the amount of backoff that is needed. From (a)-(d), the average input power $\mathcal{P}_{i 4}>\mathcal{P}_{i 3}>\mathcal{P}_{i 2}>\mathcal{P}_{i 1}$.

the baseband PA output by $y(t)$, then $\mathcal{P}_{\text {sat }}$ is the maximum output power that the PA is capable of producing, that is, $\mathcal{P}_{\text {sat }}=\max _{t}|y(t)|^{2}$. Denote by $\mathcal{P}_{m}$ the maximum input power, that is, $\mathcal{P}_{m}=\max _{t}|x(t)|^{2}$, and by $\mathcal{P}_{i}$ the average input power, that is, $\mathcal{P}_{i}=E\left[|x(t)|^{2}\right]$. The peak-to-average power ratio (PAR) is a characteristic of the input signal and is defined as $\operatorname{PAR}(s(t))=\mathcal{P}_{m} / \mathcal{P}_{i}[11]$ or PAR $(\mathrm{dB})=\mathcal{P}_{m}(\mathrm{~dB})-$ $\mathcal{P}_{i}(\mathrm{~dB})$.

For a given $\mathcal{P}_{\text {sat }}$ and gain of the PA, the efficiency of the PA increases with increasing $\mathcal{P}_{i}$. In Figure $1(\mathrm{a})$, the PA is linear up to $\mathcal{P}_{m 1}$, but is nonlinear afterwards. Nonlinearity generates in-band distortion as well as adjacent channel interference. To avoid these detrimental nonlinear effects, the input signal is often backed-off to the PA's linear region as shown in Figure 1(a). The corresponding power efficiency is very low, often in the range of $10 \%$ or much less [9]. With PA linearization, we strive to achieve an ideal linear input-output characteristic shown in Figure 1(b). The input signal is amplified undistorted until $\mathcal{P}_{\text {sat }}$ is reached. In Figure $1(\mathrm{~b})$, the average input power is higher than that in Figure 1(a), that is, $\mathcal{P}_{i 2}>\mathcal{P}_{i 1}$, demonstrating how power efficiency can be improved via PA linearization. If we can reduce the PAR of the input signal as well, we arrive at a situation depicted in Figure 1(c). The peak power is the same as in Figure 1(b), but thanks to PAR reduction, the average input power is increased, that is, $\mathcal{P}_{i 3}>\mathcal{P}_{i 2}$, further boosting the efficiency of the PA. If we drive the PA harder by scaling up the input so the signal occasionally enters the saturation region of the PA (see Figure 1(d)), we can achieve even higher efficiency at the expense of controllable nonlinear distortions.

In this paper, we explain by theoretical analysis and demonstrate by testbed experiments how the combination of PAR reduction and PA linearization can significantly improve the transmission power efficiency. PA linearization usually functions regardless of the input signal format (e.g., OFDM versus CDMA), but many PAR reduction algorithms are developed with a particular type of signal in mind. In this paper, we will focus on the OFDM signal when we investigate the PAR reduction method, but the proposed technique can be modified for other signal formats such as CDMA as well. 


\section{PAR REDUCTION}

\subsection{Threshold on PAR}

Denote by $\left\{S_{l}[k]\right\}_{k=0}^{N-1}$ the $l$ th block of the frequency-domain OFDM signal drawn from a known constellation, where $N$ is the number of subcarriers. For the rest of the paper, we will drop the block index $l$ for notational simplicity, since OFDM can be free of interblock interference with proper use of the cyclic prefix. The corresponding time-domain signal is $s(t)=(1 / \sqrt{N}) \sum_{k=0}^{N-1} S[k] e^{j 2 \pi k t / T_{s}}, 0 \leq t \leq T_{s}$, where $T_{s}$ is the OFDM symbol period and $j=\sqrt{-1}$.

The worst possible PAR of an OFDM signal is $N$ (e.g., when $S[k]$ is the same for each $k$ ). To amplify $s(t)$ absolutely without any distortion, we need to position the highest possible peak power at $\mathcal{P}_{m 2}$ in Figure 1(b). Under this arrangement, the average power $\mathcal{P}_{i 2}$ and thus the PA efficiency will be very low.

In practice, a PA is expected to provide a certain level of power efficiency, which means that for a given PA and biasing conditions, the average input power $\mathcal{P}_{i}$ has to be above a certain amount. This also requires the input signal PAR to be less than a threshold $\gamma_{0}$. The concept of PAR thresholding was also explored in [10] for the partial transmit sequence technique.

\subsection{Review of selected mapping for OFDM}

The complementary cumulative distribution function (CCDF) of the PAR of the continuous-time $s(t)$ was suggested in [12]

$$
\operatorname{Pr}\{\operatorname{PAR}(s(t))>\gamma\}=1-\exp \left\{-e^{-\gamma} N \sqrt{\frac{\pi}{3} \ln N}\right\} .
$$

Selected mapping (SLM) was first proposed in [5] as a distortionless technique to reduce the PAR of OFDM signals. Assume that an i.i.d. phase table $\left\{\phi^{(m)}[k]\right\}_{0 \leq k \leq N-1}^{1 \leq m \leq M}$ is available at the transmitter and at the receiver. Let us first rotate the phases of $S[k]$ to obtain $S^{(m)}[k]=S[k] e^{j \phi^{(m)}[k]}$. From among the $M$ equivalent time-domain representations, $\left\{s^{(m)}(t)\right\}_{m=1}^{M}, s^{(\bar{m})}(t)$, which has the lowest PAR, is transmitted, that is, $\operatorname{PAR}\left(s^{(\bar{m})}(t)\right)=\min _{1 \leq m \leq M} \operatorname{PAR}\left(s^{(m)}(t)\right)$.

Optimal design of the phase table $\left\{\phi^{(m)}[k]\right\}_{0 \leq k \leq N-1}^{1 \leq m \leq M}$ has been investigated in [13]: the PAR reducing capability of SLM is maximized when $\left\{\phi^{(m)}[k]\right\}$ are i.i.d. satisfying $E\left[e^{j \phi^{(m)}[k]}\right]=0$. Under this optimality condition, the timedomain signals $s^{(m)}(t)$ and $s^{(l)}(t)$ can be shown to be asymptotically independent for $m \neq l$. Consequently, for a large $N$, we can obtain the CCDF of the SLM-OFDM signal $s^{(\bar{m})}(t)$ as follows:

$$
\operatorname{Pr}\left\{\operatorname{PAR}\left(s^{(\bar{m})}(t)\right)>\gamma\right\}=[1-a]^{M},
$$

where $a=\exp \left\{-e^{-\gamma} N \sqrt{(\pi / 3) \ln N}\right\}$ (cf. (1)).

We make the following remarks regarding the "conventional" SLM described above.
(1) SLM aims at minimizing the PAR per OFDM block by carrying out all $M$ mappings. Even if the first few mappings have already managed to reduce the PAR to be below a certain threshold $\gamma_{0}$, the SLM scheme still continues to seek further reduction of the PAR.

(2) For given $N$ and $\gamma_{0}$ values, (2) shows that even after all $M$ mappings are tried out, there is still a nonzero probability that the SLM method fails to meet the PAR goal, that is, the resulting $\operatorname{PAR}\left(s^{(\bar{m})}(t)\right)>\gamma_{0}$. When that happens, $s^{(\bar{m})}(t)$ will need to be clipped to meet the peak power and average power constraints.

(3) For given $N$ and $M$ values and clipping probability $p=\operatorname{Pr}\left\{\operatorname{PAR}\left(s^{(\bar{m})}(t)\right)>\gamma_{0}\right\}$, we can find from (2) the corresponding PAR threshold

$$
\gamma_{0}=\ln \left(N \sqrt{\frac{\pi}{3} \ln N}\right)-\ln \left(\ln \frac{1}{1-p^{1 / M}}\right)
$$

We investigate next a modified SLM technique which incorporates the above PAR thresholding and clipping considerations.

\subsection{SLM with thresholding and clipping}

Our objective here is to apply SLM, but to stop trying as soon as the PAR threshold $\gamma_{0}$ is met, with the constraint that the number of trials is no more than $M$ (including the original OFDM signal). Our strategy is "to do only what is necessary" in order to save computational resources. As mentioned before, there is always the possibility that even after all $M$ trials, SLM still fails to meet the PAR goal $\gamma_{0}$. In that case, $s^{(\bar{m})}(t)$ is clipped to become $x(t)$, which has maximum amplitude $\sqrt{\mathcal{P}_{i} \gamma_{0}}$ (the clipping level). As long as the clipping probability (2) evaluated at $\gamma_{0}$ is small (e.g., $10^{-3}$ ), there will be negligible amount of spectral regrowth or BER increase.

The step-by-step algorithm for the proposed SLM with thresholding and clipping (SLMTC) technique is described in Algorithm 1.

In [14], SLM was proposed to reduce the PAR of the forward link CDMA signal using random phase and PN offset mapping. The concept of thresholding and clipping described above is not restricted to any specific signal format; for example, it can be applied to the CDMA system as well.

We note that combining SLM with threshold clipping is not merely doing both; the SLM algorithm exits if the predetermined PAR threshold is met. PA linearization operates independently of PAR reduction however, as we elaborated in Section 2.

\subsection{Performance analysis of SLMTC}

We analyze here the CCDF expression for the PAR of the SLMTC signal $x(t)$ obtained as described in the previous section. Denote by $s^{(\bar{m})}(t)$ the signal after SLM with thresholding, which is not to be confused with the $s^{(\bar{m})}(t)$ notation used in the conventional SLM (cf. Section 3.2). If $\gamma \leq \gamma_{0}$, the event $\operatorname{PAR}(x(t)) \leq \gamma$ is equivalent to the event $\operatorname{PAR}\left(s^{(\bar{m})}(t)\right) \leq \gamma$, 
Step 1. Set $\bar{m}=m=1$.

Step 2. Form $s^{(m)}(t)$ and compute $\operatorname{PAR}\left(s^{(m)}(t)\right)$.

Step 3. If $\operatorname{PAR}\left(s^{(m)}(t)\right) \leq \gamma_{0}$, then continue to Step 4; else go to Step 5.

Step 4. Set $\bar{m}=m$ and $x(t)=s^{(\bar{m})}(t)$, and go to Step 8 .

Step 5. If $\operatorname{PAR}\left(s^{(m)}(t)\right)<\operatorname{PAR}\left(s^{(\bar{m})}(t)\right)$, then go to Step 5.1; else go to Step 5.2.

Step 5.1. Set $\bar{m}=m$.

Step 5.2. $m=m+1$.

Step 6. If $m>M$, then go to Step 7; else go to Step 2 .

Step 7. $\operatorname{Clip} s^{(\bar{m})}(t)$ to form $\left(A=\sqrt{\mathcal{P}_{i} \gamma_{0}}\right)$

$$
x(t)= \begin{cases}s^{(\bar{m})}(t) & \text { if }\left|s^{(\bar{m})}(t)\right| \leq A, \\ A \exp \left\{j \angle s^{(\bar{m})}(t)\right\} & \text { otherwise. }\end{cases}
$$

Step 8. Transmit $x(t)$.

Algorithm 1: SLM with thresholding and clipping.

which in turn is equivalent to the event

$$
\begin{array}{r}
\exists 1 \leq d \leq M, \quad \text { such that } \operatorname{PAR}\left(s^{(d)}(t)\right) \leq \gamma, \\
\left\{\operatorname{PAR}\left(s^{(l)}(t)>\gamma_{0}\right)\right\}_{l=1}^{d-1} .
\end{array}
$$

By recalling (1), we obtain

$$
\begin{aligned}
\operatorname{Pr} & \{\operatorname{PAR}(x(t)) \leq \gamma\} \\
& =\sum_{d=1}^{M} \operatorname{Pr}\left\{\operatorname{PAR}\left(s^{(d)}(t)\right) \leq \gamma\right\} \prod_{l=1}^{d-1} \operatorname{Pr}\left\{\operatorname{PAR}\left(s^{(l)}(t)\right)>\gamma_{0}\right\} \\
& =\sum_{d=1}^{M} a\left(1-a_{0}\right)^{d-1}=\frac{a}{a_{0}}\left(1-\left(1-a_{0}\right)^{M}\right), \quad \text { for } \gamma \leq \gamma_{0},
\end{aligned}
$$

where $a_{0}=\exp \left\{-e^{-\gamma_{0}} N \sqrt{(\pi / 3) \ln N}\right\}$.

Obviously due to clipping,

$$
\operatorname{Pr}\{\operatorname{PAR}(x(t))>\gamma\}=0, \quad \text { for } \gamma>\gamma_{0} .
$$

Combining (6) and (7), we find the CCDF of the PAR for the proposed SLMTC method:

$$
\operatorname{Pr}\{\operatorname{PAR}(x(t))>\gamma\}= \begin{cases}1-\frac{a}{a_{0}}\left[1-\left(1-a_{0}\right)^{M}\right], & \gamma \leq \gamma_{0}, \\ 0, & \gamma>\gamma_{0} .\end{cases}
$$

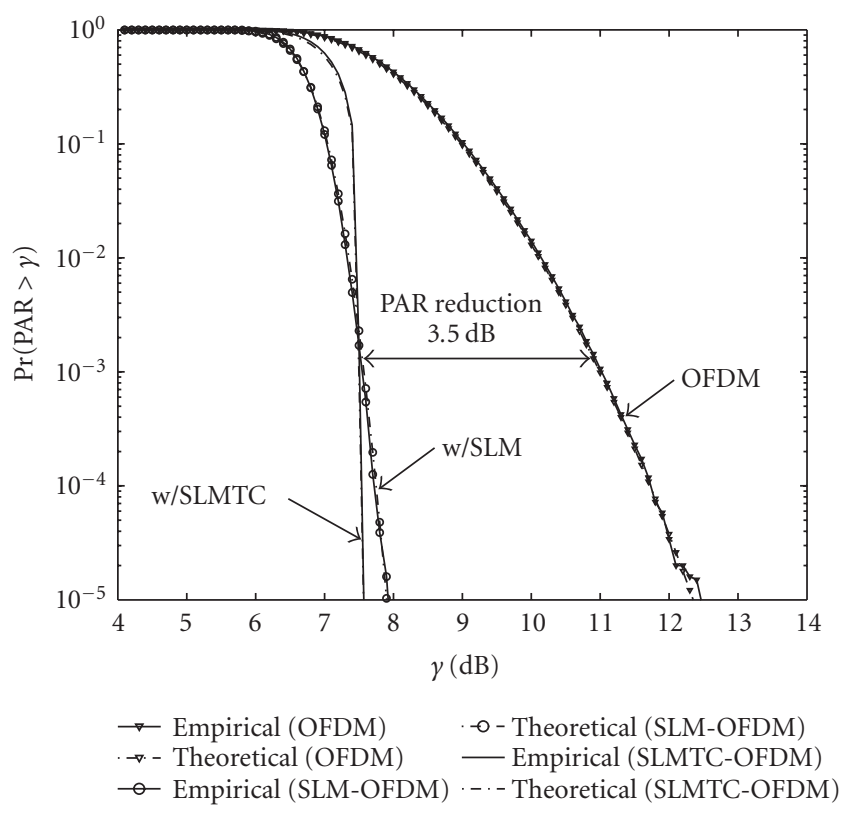

FIGURE 2: CCDF of the PAR for the OFDM signal, OFDM signal with SLM, and OFDM signal with SLMTC.

\subsection{Validation of the CCDF expressions}

In the computer simulations, the number of subcarriers $N=128$, the maximum number of phase rotations $M=$ 16 , and the PAR threshold $\gamma_{0}=7.5 \mathrm{~dB}$. The frequencydomain OFDM subsymbols were drawn independently from a QPSK constellation, and $10^{6}$ Monte Carlo runs were performed. Figure 2 shows the empirical CCDFs (solid lines) of $\operatorname{PAR}(s(t))(\mathrm{OFDM}), \operatorname{PAR}\left(s^{(\bar{m})}(t)\right)(\mathrm{SLM})$, and $\operatorname{PAR}(x(t))$ (SLMTC), along with the corresponding theoretical CCDFs (dash-dotted lines) calculated from (1), (2), and (8), respectively. The empirical CCDFs of the continuous-time PAR were obtained by evaluating the discrete-time PAR of the 4-time oversampled OFDM signal [11]. It is evident from Figure 2 that the theoretical and the empirical CCDFs agreed very well. We observe that when $M=16$, the proposed algorithm achieved $3.5 \mathrm{~dB}$ of PAR reduction at the CCDF level of $10^{-3}$. Indeed, if we substitute $N=128$ and $p=10^{-3}$ into (1), we obtain $\gamma=12.5720 \cong 11(\mathrm{~dB})$; if we substitute $N=128, M=16$, and $p=10^{-3}$ into (3), we obtain $\gamma_{0}=5.6178 \cong 7.5(\mathrm{~dB})$. Thus, PAR reduction in the amount of $\gamma-\gamma_{0}=3.5 \mathrm{~dB}$ was achieved at the CCDF level of $p=10^{-3}$.

We observe from Figure 2 that the CCDF curves for SLM and SLMTC cross over at $\gamma_{0}$, and SLMTC has less PAR reducing capability than SLM for $\gamma<\gamma_{0}$. This is completely expected since by design, SLMTC generally uses fewer mappings and consumes less computational resources than SLM. Unless one pursues block-by-block adaptive biasing or linear scaling [15] approaches, any PAR value lower than the required $\gamma_{0}$ does not necessarily lead to additional power savings. We regard SLMTC as a lower-cost alternative to SLM. As we mentioned in Section 3.3, the resources savings from the 


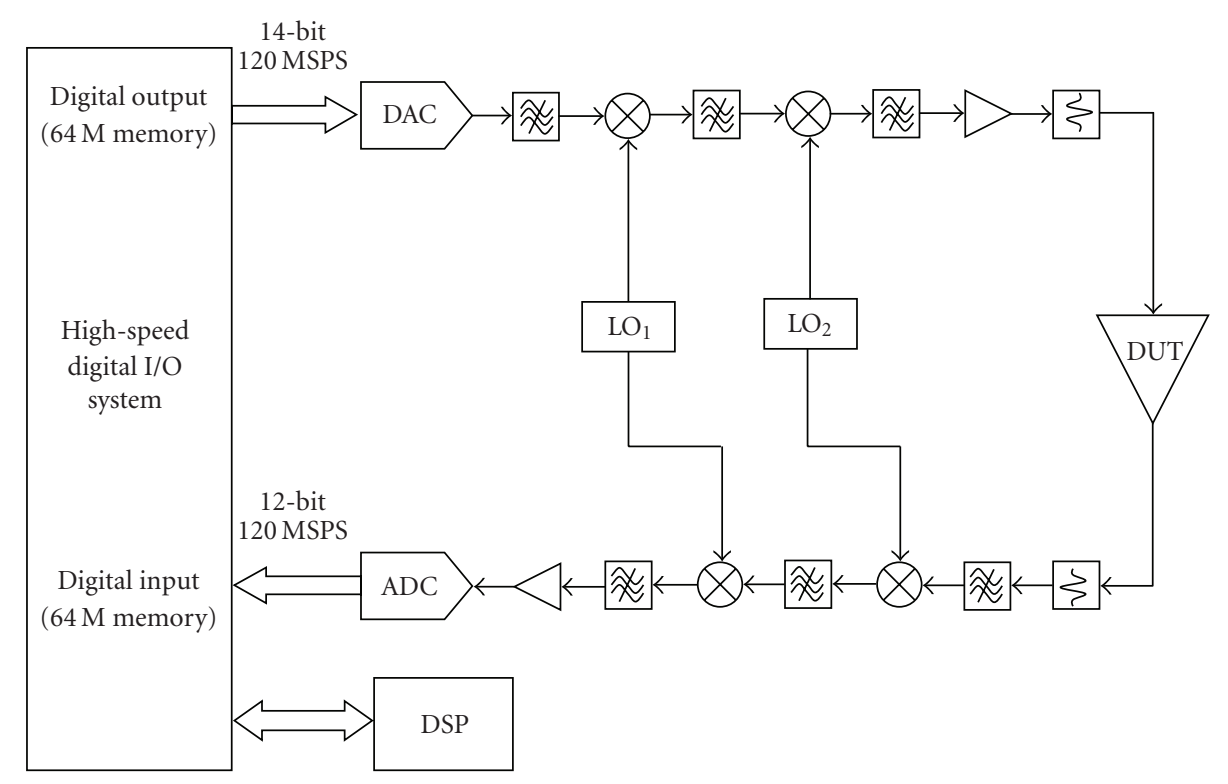

FIgURE 3: Block diagram of the testbed.

PAR thresholding can be harvested using a buffered dynamic processing scheme [16], which results in a smaller transmission latency than SLM, and thus permits a higher data rate.

\section{DIGITAL BASEBAND PREDISTORTION LINEARIZATION OF THE PA}

We adopt the memory polynomial predistorter (PD) model given by [6]

$$
z[n]=\sum_{k=1}^{K} \sum_{q=0}^{Q} a_{k q} x[n-q]|x[n-q]|^{k-1},
$$

where $x[n]=\left.x(t)\right|_{t=n / F_{s}}$ is the sampled version of the input $x(t)$ with sampling frequency $F_{s}, z[n]$ is the discrete-time output of the PD, and $\left\{a_{k q}\right\}$ are the PD coefficients. This PD has memory depth $Q$ and highest nonlinearity order $K$. The indirect learning architecture is used to solve for the parameters $\left\{a_{k q}\right\}$ via linear least squares; see [6] for details. Note that when $Q=0,(9)$ becomes a memoryless polynomial PD, which may be sufficient for memoryless PAs, such as handset PAs with narrowband inputs.

\section{TESTBED EXPERIMENTS}

We have conducted testbed experiments on two different PAs to demonstrate our approach. Our goal is to show that for the same PA, it is possible to boost the average transmit power through PAR reduction and PA linearization, while keeping the ACPR unchanged.

Figure 3 depicts the configuration of the testbed, which consists of a high-speed digital I/O system, a digital-toanalog converter (DAC), RF transmit and receive chains, a device under test (DUT), and an analog-to-digital converter (ADC). The high-speed digital I/O system has 150 million samples per second (MSPS), 16-bit digital input/output capability. In the transmission mode, the digital I/O system first generates baseband data, applies the SLMTC algorithm, predistorts it, and then digitally upconverts the signal to an intermediate frequency (IF) of $30 \mathrm{MHz}$, and finally sends out the 14-bit data stream to the DAC at a sampling rate of 120 MSPS. Superheterodyne upconversion and downconversion chains are used to convert the digital IF signal to and from the carrier frequency. The DUTs are, respectively, a $1 \mathrm{~W}$ handset PA and a $45 \mathrm{~W}$ base-station PA. In the acquisition mode, the digital I/O system acquires 12-bit digital IF data at the sampling rate of 120 MSPS from the ADC. The received baseband data $y[n]$ is obtained by converting the PA output to baseband and removing the time delay between the input and the output of the digital I/O system. Since the signal is modulated in the digital domain, any inphase and quadrature imbalance problem in the quadrature modulator is obviated.

\subsection{Experiment on the $1 \mathrm{~W}$ handset PA}

In this experiment, the DUT is the $1 \mathrm{~W}$ handset PA. The input is an OFDM signal centered at $836 \mathrm{MHz}$ with a $1.25 \mathrm{MHz}$ bandwidth and 128 subcarriers. We measured the power spectral density (PSD) of the PA output using a spectrum analyzer. ACPR was measured as the ratio between the average power in the adjacent channel and the average power in the main channel, both over a $30 \mathrm{KHz}$ bandwidth [9]. The requirement was to keep the ACPR below $-50 \mathrm{dBc}$. Figure 4 shows the PSDs of the PA output when (a) the input was backedoff just enough to meet the ACPR requirement; (b) a memoryless polynomial PD (i.e., $Q=0, K=5$ in (9)) was applied, and the amount of input backoff was reduced; (c) both SLMTC $\left(M=16, \gamma_{0}=7.5 \mathrm{~dB}\right)$ and the memoryless polynomial PD were applied, requiring even less input 


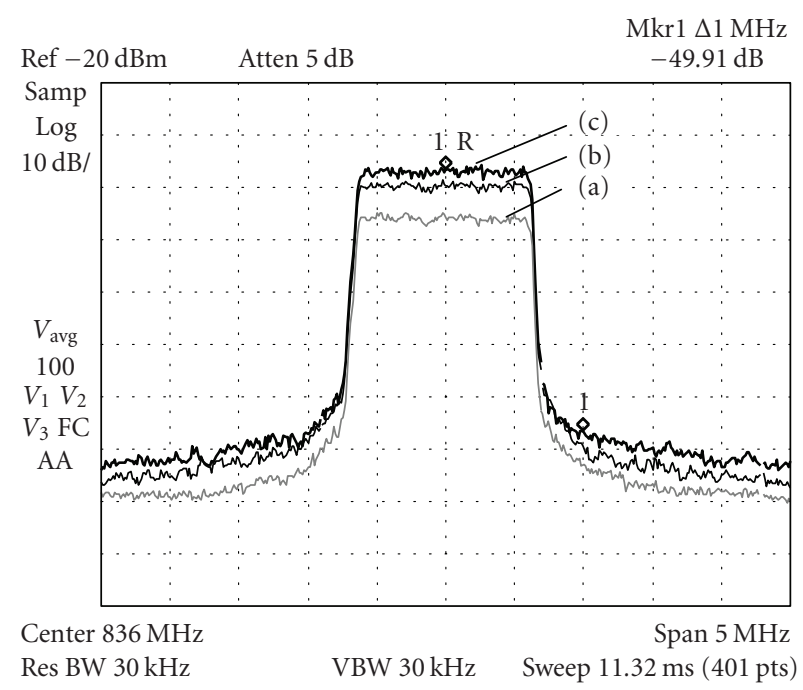

Figure 4: Power spectral density measurements at the output of the $1 \mathrm{~W}$ handset PA when (a) the input was backed-off, (b) a memoryless polynomial $\mathrm{PD}(Q=0, K=5)$ was applied, and (c) both SLMTC $\left(M=16, \gamma_{0}=7.5 \mathrm{~dB}\right)$ and the memoryless polynomial PD $(Q=0, K=5)$ were applied.

backoff. By comparing curves (a) and (b) in Figure 4, we see that the average output power in the main channel increased by $6 \mathrm{~dB}$ thanks to the use of the PD and the resulting reduction in backoff. Moreover, with the SLMTC PAR reduction technique, we were able to boost the average output power by another $3 \mathrm{~dB}$ without introducing any spectral regrowth (cf. lines (b) and (c)). Therefore, we have achieved a total of $9 \mathrm{~dB}$ increase in the average output power of the PA through the combination of PAR reduction and predistortion linearization.

\subsection{Experiment on the $45 \mathrm{~W}$ base-station $P A$}

In this experiment, the DUT is the $45 \mathrm{~W}$ base-station PA. The input is an OFDM signal centered at $881 \mathrm{MHz}$ with a $2.5 \mathrm{MHz}$ bandwidth and 128 subcarriers. For the $45 \mathrm{~W}$ $\mathrm{PA}$, the requirement was to keep the ACPR below $-45 \mathrm{dBc}$. Figure 5 shows the PSDs of the PA output when (a) the input was backed-off just enough to meet the ACPR specification; (b) a memory polynomial PD (i.e., $Q=5, K=$ 5 in (9)) was applied; (c) both $\operatorname{SLMTC~}\left(M=16, \gamma_{0}=\right.$ $7.5 \mathrm{~dB}$ ) and the memory polynomial PD were applied. From Figure 5, we can see that the average output power was increased by $11 \mathrm{~dB}$ through the combination of PAR reduction and predistortion linearization. Through experimentation, we have found that this high power amplifier had significant memory effects and that memoryless predistortion was not as effective as the memory polynomial predistortion demonstrated here.

\section{CONCLUSIONS}

We proposed in this paper joint PAR reduction and PA linearization as an effective approach to improve the efficiency

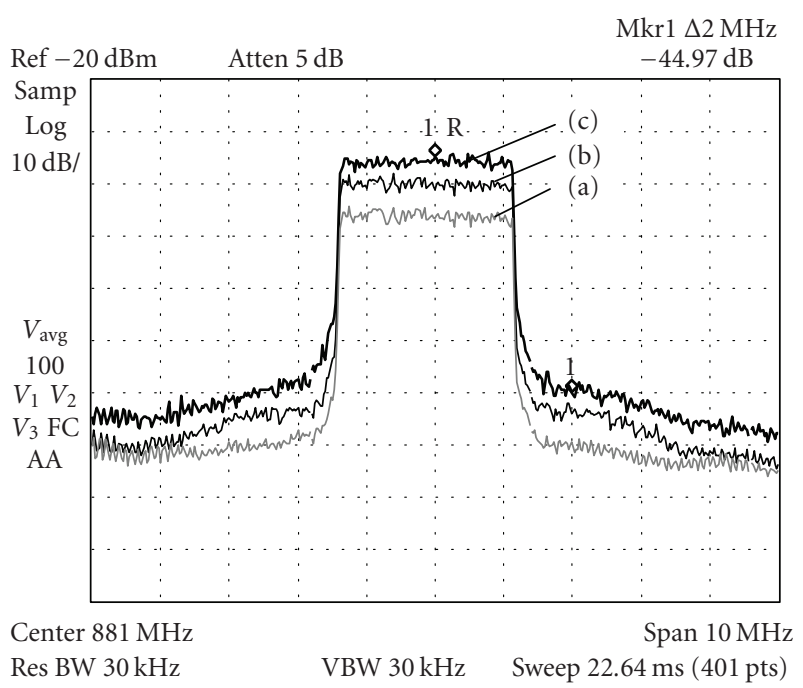

FIGURe 5: Power spectral density measurements at the output of the $45 \mathrm{~W}$ base-station PA when (a) the input was backed-off, (b) a memory polynomial $\mathrm{PD}(Q=5, K=5)$ was applied, and (c) both SLMTC $\left(M=16, \gamma_{0}=7.5 \mathrm{~dB}\right)$ and the memory polynomial $\mathrm{PD}(Q=5, K=5)$ were applied.

of the RF/microwave PA in wireless communications. For PAR reduction, we discussed a thresholding and clipping technique to reduce the computational resource requirements of selected mapping (SLM). A closed-form CCDF expression was derived for the resulting PAR. For PA linearization, we adopted the (memory) polynomial predistorter for its simplicity and robustness. PAR reduction and PA linearization can be applied independently, so many combinations of PAR reduction and PA linearization techniques may work. Using testbed experiments, we demonstrated the effectiveness of our technique as significant increase in the average output power without exceeding the spectral emission limits. Our analysis uses OFDM as the model system, but the idea of joint PAR reduction and PA linearization applies to other systems characterized by high PAR values as well.

\section{ACKNOWLEDGMENTS}

The authors would like to thank Mr. Robert J. Baxley for insightful discussions on the PAR thresholding idea. This work was supported in part by the US National Science Foundation Grants 0218778 and 0219262, the US Army Research Laboratory Communications and Networks Collaborative Technology Alliance Program, and the Texas Instruments DSP Leadership University Program.

\section{REFERENCES}

[1] A. A. Triolo, "Advanced techniques in high-efficiency power amplification for WCDMA," in NJIT ECE Seminar, February 24, 2003.

[2] "Digitally controlled transmitters for next generation communications systems," http://www-cwc.ucsd.edu/rp_03-05_I.php. 
[3] S. M. Ju and S. H. Leung, "Clipping on COFDM with phase on demand," IEEE Communications Letters, vol. 7, no. 2, pp. 49-51, 2003.

[4] A. E. Jones, T. A. Wilkinson, and S. K. Barton, "Block coding scheme for reduction of peak to mean envelope power ratio of multicarrier transmission schemes," Electronics Letters, vol. 30, no. 25, pp. 2098-2099, 1994.

[5] R. W. Bäuml, R. F. H. Fischer, and J. B. Huber, "Reducing the peak-to-average power ratio of multicarrier modulation by selected mapping," Electronics Letters, vol. 32, no. 22, pp. 20562057, 1996.

[6] L. Ding, G. T. Zhou, D. R. Morgan, et al., "A robust digital baseband predistorter constructed using memory polynomials," IEEE Transactions on Communications, vol. 52, no. 1, pp. 159-165, 2004.

[7] H.-G. Ryu, T. P. Hoa, K. M. Lee, S.-W. Kim, and J.-S. Park, "Improvement of power efficiency of HPA by the PARR reduction and predistortion," IEEE Transactions on Consumer Electronics, vol. 50, no. 1, pp. 119-124, 2004.

[8] R. Sperlich, Y. Park, G. Copeland, and J. S. Kenney, "Power amplifier linearization with digital pre-distortion and crest factor reduction," in Proceedings of IEEE MTT-S International Microwave Symposium Digest, vol. 2, pp. 669-672, Fort Worth, Tex, USA, June 2004.

[9] S. C. Cripps, RF Power Amplifiers for Wireless Communications, Artech House, Boston, Mass, USA, 1999.

[10] A. D. S. Jayalath and C. Tellambura, "Adaptive PTS approach for reduction of peak-to-average power ratio of OFDM signal," Electronics Letters, vol. 36, no. 14, pp. 1226-1228, 2000.

[11] J. Tellado, Multicarrier Modulation with Low PAR-Applications to DSL and Wireless, Kluwer Academic, Dordrecht, The Netherlands, 2000.

[12] S. Wei, D. L. Goeckel, and P. A. Kelly, "The complex envelope of bandlimited OFDM signals is asymptotically Gaussian: proof and application," http://www.ece.lsu.edu/swei.

[13] G. T. Zhou and L. Peng, "Optimality condition for selected mapping in OFDM," IEEE Transactions on Signal Processing, vol. 54, no. 8, pp. 3159-3165, 2006.

[14] N. Chen and G. T. Zhou, "Distortionless crest factor reduction for forward link CDMA," in Proceedings of the 6th IEEE Workshop on Signal Processing Advances in Wireless Communications (SPAWC'05), pp. 294-297, New York, NY, USA, June 2005.

[15] H. Ochiai, "Performance analysis of peak power and bandlimited OFDM system with linear scaling," IEEE Transactions on Wireless Communications, vol. 2, no. 5, pp. 1055-1065, 2003.

[16] H. Qian, C. Xiao, N. Chen, and G. T. Zhou, "Dynamic selected mapping for OFDM," in Proceedings IEEE International Conference on Acoustics, Speech and Signal Processing (ICASSP '05), vol. 4, pp. 325-328, Philadelphia, Pa, USA, March 2005.

Ning Chen received his dual B.S. degrees in electronic engineering and in accounting from the Shanghai Jiao Tong University (SJTU), China, in July 1997. He worked as an Instructor at SJTU until August 2000. He received his M.S. degree in electrical and computer engineering from the New Mexico State University in December 2001. He earned the Ph.D. degree in electrical engineering from the Georgia Institute of

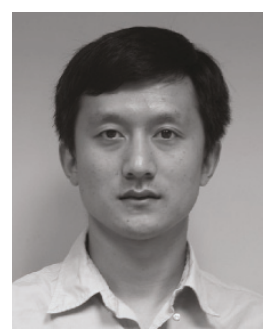

Technology, Atlanta, in 2006. He is currently employed by Freescale Semiconductor, Inc., in Austin, Tx, USA. His general research interests are in the areas of signal processing and communications. Specific current interests include predistortion linearization of nonlinear power amplifiers, peak-to-average power ratio reduction of communication signals, communication channel identification and equalization, and adaptive algorithm development on DSP.

G. Tong Zhou received her B.S. degree in biomedical engineering and instrumentation from the Tianjin University, China, in July 1989. From September 1989 to May 1995, she was with the University of Virginia (UVA), where she obtained her M.S. degree in biophysics in May 1992, M.S. degree in electrical engineering in January 1993, and Ph.D. degree in electrical engineering in January 1995. She has been with

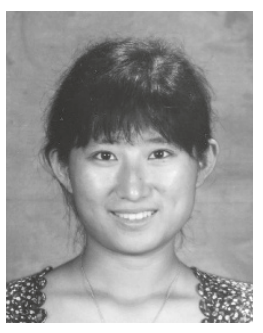
the School of Electrical and Computer Engineering at Georgia Tech since September 1995 where she is now a Professor. In 1997, she received the National Science Foundation Faculty Early Career Development (CAREER) Award. She is also recipient of the 2000 Meritor Teaching Excellence Award at Georgia Tech. Her research interests are in the general areas of statistical signal processing and communications applications.

Hua Qian received his B.S. and M.S. degrees in electrical engineering from Tsinghua University, Beijing, China, in 1998 and 2000, respectively. He received the Ph.D. degree in electrical and computer engineering from the Georgia Institute of Technology, Atlanta, Ga, USA, in 2005. He is currently a Senior Design Engineer at Marvell Semiconductor Inc. His general research interests are in the areas of signal process-

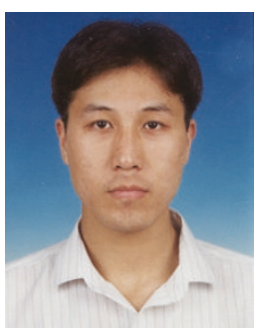
ing and communications. Specific current interests include studying nonlinear effects in wireless communication systems, such as digital baseband predistortion linearization for power amplifiers with memory effects and peak-to-average power ratio reduction for wireless transmissions. 